\title{
STIMULUS TO CONVERGENCE IN NORMAL AND ASTHENOPIC SUBJECTS*
}

\author{
BY
}

\author{
H. ASHER
}

Physiology Department, University of Birmingham

A new type of stereoscope has recently been described (Asher and Law, 1952) in which the lateral separation of the cards and their distance from the lenses may both be varied separately, thus exercising accommodation and convergence independently of each other. It was expected that when the images of the cards were at infinity, the normal orthophoric observer would find fusion easiest with the visual axes parallel. However, it was at once apparent that this was not so, since the majority of observers found it easier to combine the pictures with the visual axes converged by as much as $10^{\circ}$ or even more, and quite difficult to combine them with the visual

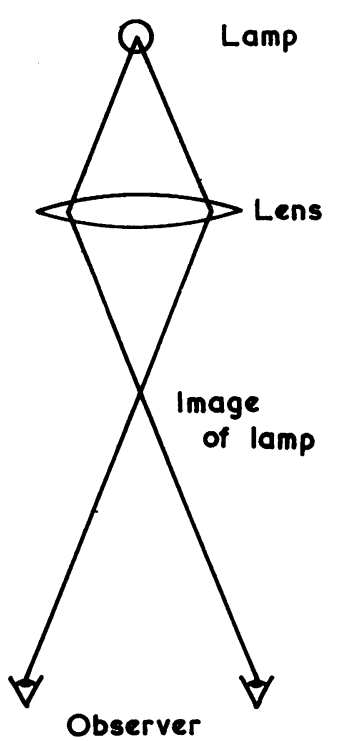

FIG. 1.-Vergence and disparity clues from the real image of the lamp give information that the lamp is on the near side; nevertheless it appears at first to be on the far side. This is because the real image of the lamp does not obstruct the view of anything behind it. axes parallel. Even patients with a pronounced exophoria would set the instrument so as to combine the images in an over-converged position.

Enquiry showed that this effect is familiar to many who use instruments of this kind. Hofstetter (1942), Morgan (1944), and Ittelson and Ames (1950) have shown that awareness of the position of the object (the proximal factor) can affect both accommodation and convergence. It is the purpose of this paper to demonstrate the extent to which the usual clinical tests, such as the Maddox rod and the Maddox wing, are influenced by the proximal factor; then to study the role played by this factor in determining convergence both in normal and asthenopic subjects; and finally to indicate the bearing of the results upon the diagnosis of asthenopia and upon the amount of correction advisable in presbyopia and hypermetropia.

The importance of the proximal factor may be shown by the following very simple experiment (Fig. 1).

A small lamp is placed at about $20 \mathrm{~cm}$. from a $+10 \mathrm{D}$ spherical lens, so that a real inverted image of the lamp is formed at a distance of $20 \mathrm{~cm}$. from the lens and on the

* Received for publication June 25, 1952. 
other side of it. The observer places his head about $30 \mathrm{~cm}$. from this image, and by closing each eye in turn makes sure that each does in fact receive light from it. With both eyes open he now sees two lamps, somewhat out of focus and apparently situated on the far side of the lens. If a pin is placed by the real image and fixated, the lamp is immediately seen as single and clearly focused. After a while a pleasantly vivid sense of the position of the image in space develops, and this may even cause the observer to cry out with pleasure. The impression can now be maintained when the pin is removed.

It is clear that the eyes converge and accommodate to the point on the far side of the lens where the light is thought to be. It is thought to be there because the image does not obstruct the vision of any part of the view on the near side, as would a real object in this position. Both the vergence of the light rays and the disparity between the retinal images give information that the image is on the near side, so that with a little practice the image may be seen in its true position. What is of interest is that, for the unpractised observer, the 'common-sense estimate' of the position of the lamp, based chiefly on absence of obstruction, will outweigh the other clues.

The following investigation was carried out to find more precisely the role played by the 'commonsense estimate' in determining convergence and accommodation, both in normal observers and in those with convergence defects.

\section{Material}

Tests were made with 22 normal observers; for the purposes of the tests a person was classed as normal if he had no symptoms of eye strain, irrespective of whether he had a latent squint or not. All were volunteers from the Birmingham Medical School, and they were mostly medical students in their early twenties, though a few older people were also included. Thirteen patients having symptoms thought to be associated with convergence defects were also tested; of these, eleven were attending for treatment at the Orthoptic Department of the Birmingham and Midland Eye Hospital, and two were found among the volunteers from the Medical School. They included cases of esophoria, exophoria, and convergence insufficiency.

\section{Tests}

Test 1. (See keys to Figs 2 and 3)

Any refractive error is corrected by lenses placed in a trial frame and correctly centred. The normal set-up for a Maddox rod test is used. The rod is placed in front of the left eye and the usual scale with the light in the centre is placcd on the wall at $6 \mathrm{~m}$. from the observer. Concave lenses of $-1 \mathrm{D},-2 \mathrm{D},-3 \mathrm{D}$, etc., are now placed in succession in front of both eyes. Consider as an example the case when a pair of $-2 \mathrm{D}$ lenses is placed in the frame. The wall may be taken as virtually at infinity, so that with the $-2 \mathrm{D}$ lenses an accommodative effort of $+2 \mathrm{D}$ is required to focus the image of the scale on the retina. This is the effort normally required for an object at $100 / 2=50 \mathrm{~cm}$. distant; in other words the concave lenses make the light rays diverge as if they originated from a scale placed $50 \mathrm{~cm}$. away from the observer, and we may say that there is an image of the scale formed in space in this position. Alternatively, using the formula $1 / v+1 / u=1 / f$ and substituting $u=\infty, f=-50 \mathrm{~cm}$. (for the $-2 \mathrm{D}$ lens), we get :

$$
1 / v=-1 / 50 \text {, or } v=-50 \mathrm{~cm} \text {., }
$$

showing that there is an image of the scale $50 \mathrm{~cm}$. in front of the observer. 

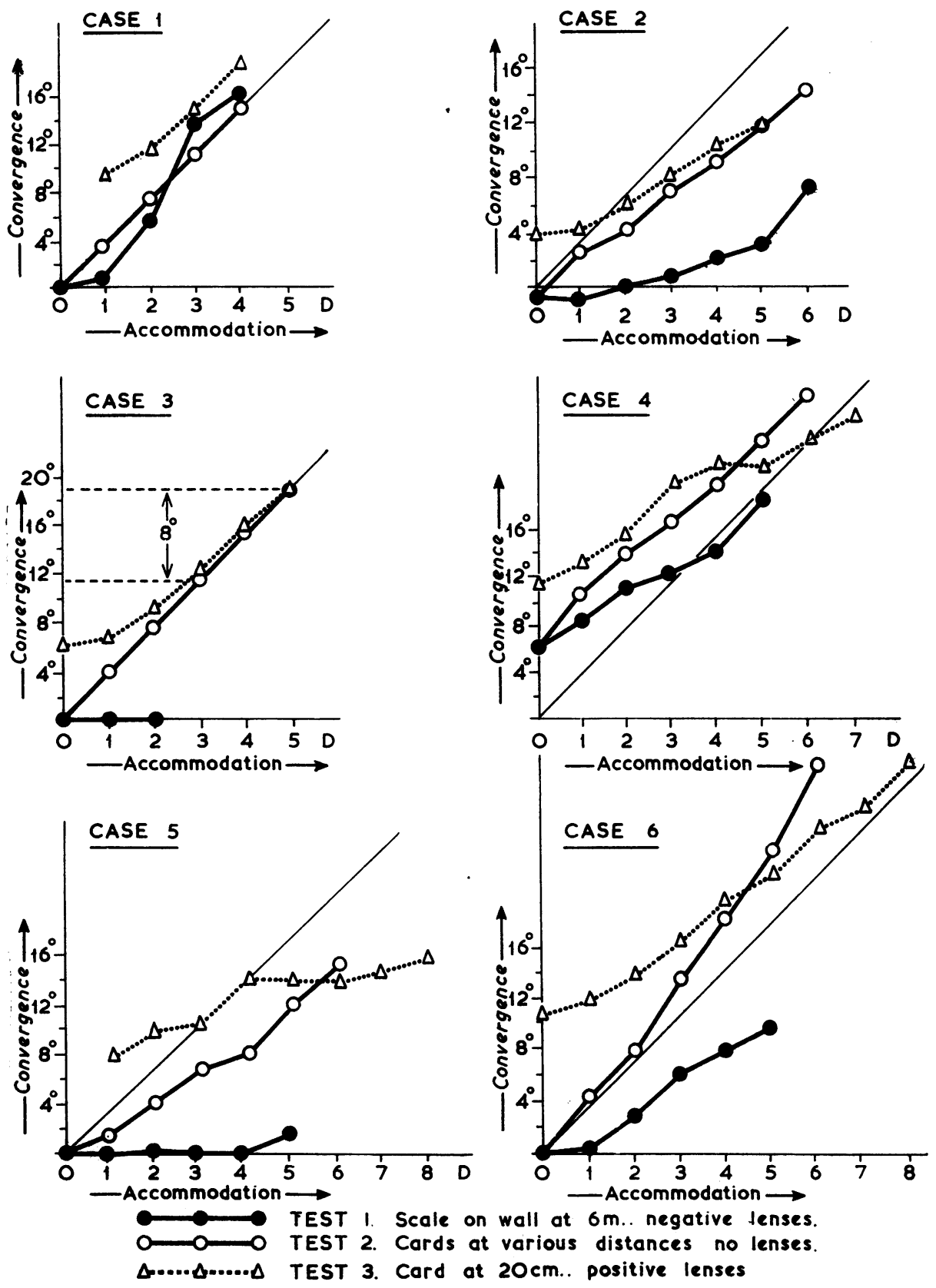

Fig. 2.-Results of Tests 1, 2, and 3 in six subjects with no symptoms. 

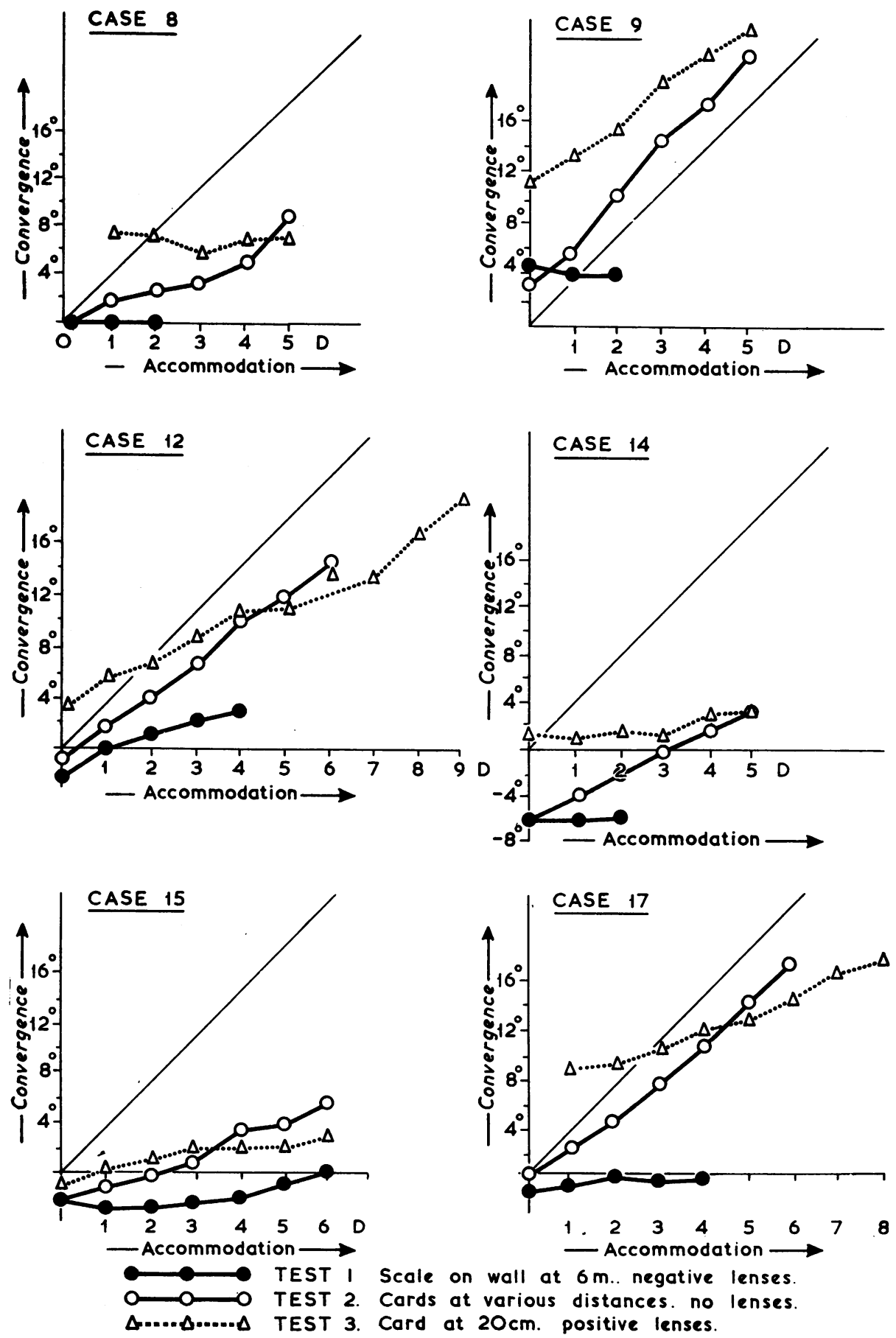

Fig. 3.-Results of Tests 1, 2, and 3 in six asthenopic patients.

Case 8-Convergence insufficiency Case 9-Esophoria

Case 12-Exophoria

Case 9-Esophoria

Case 15-Exophoria

Case 17-Convergence insufficiency 
Rays entering the eye will be in every way identical with rays which would enter the eye if there really were a scale placed in this position, of such a size that it subtended at the eye the same angle as is subtended by the wall scale. However, since this image does not obstruct the view of anything behind it, it is thought to be on the wall.

For each pair of lenses added, the patient announces the position of the red line, and in each case the visual acuity is checked by a Snellen chart placed on the wall. Readings are discontinued when vision has deteriorated by one line on the chart. A graph is now drawn in which the actual angle of convergence of the eyes is plotted against the accommodative effort. As an example, suppose that, with the rod in the left eye, when the $-2 \mathrm{D}$ lenses are added the line appears displaced by $2.5^{\circ}$ to the left, then $2.5^{\circ}$ is the actual convergence of the eyes (if, as is usual, we count $6 \mathrm{~m}$. as an infinite distance); the accommodative effort is of course $+2 \mathrm{D}$. This point is the third point on the graph of Case 6, Test 1 (Fig. 2). In many cases a concave lens was placed in front of the right eye only, as it was found that an intelligent observer could easily bring the scale sharply in focus while ignoring the lack of sharpness in the red line; this enabled the test to proceed more rapidly.

On the graph the orthophoric line is also drawn; this is a line showing the convergence appropriate to the accommodation, calculated on the basis of the measured P.D. of the patient, for an object moving in towards him from infinity. A graph which lies below this line indicates a deficit of convergence relative to accommodation (Case 6, Test 1), while a graph which is above the line shows that convergence is relatively in excess (Case 6, Test 2). It is well to avoid the terms exophoria or esophoria in connection with this test, because it would not be clear whether they indicated departures of convergence from the value required to fixate a point on the wall scale or from the values required to fixate the images of this scale. Thus, the 3rd point on the graph for Test 1 of Case 6 (Fig. 2) shows that the convergence of $2.5^{\circ}$ is excessive in relation to the (effectively) zero convergence required for the wall scale; on the other hand, it shows that the convergence is less than the value of $6.5^{\circ}$ which is indicated by the orthophoric line to be appropriate to the accommodative effort of $-2 \mathrm{D}$, and which is the amount of convergence required to fixate a point on the image of the scale formed by the $+2 \mathrm{D}$ lens.

Test 2. (See key to Figs 2 and 3)

A smaller scale marked in degrees is so made that the $1^{\circ}$ scale divisions again subtend $1^{\circ}$ at the observer's eye when placed $1 \mathrm{~m}$. from him. The scale then is placed at this distance, and a light shines through the centre of it. The Maddox rod is placed in the left eye, no lenses other than those required to correct any refractive error are worn, and the subject announces the position of the red line. If the line appears to go through the lamp it signifies that the visual axes are converged correctly upon the lamp, and as the P.D. of the observer is known, the angle of convergence may be simply calculated. For example, for a P.D. of $65 \mathrm{~mm}$., the angle between the visual axes for a point at $1 \mathrm{~m}$. will be $3.7^{\circ}$. If the observer now sees the line as situated $2^{\circ}$ to the left of the lamp, then his actual convergence will be $3.7+2=5.7^{\circ}$. Now the accommodative effort involved is 1 dioptre, and this is exactly the same as for the case when the observer wore a $-1 \mathrm{D}$ lens and looked at the scale on the wall. This can also be expressed by saying that the card is placed so as to occupy the position in which the image of 
the wall scale was situated in the corresponding part of the previous test. The amount of convergence is quite different in the two cases, because in each test the observer is fully aware of the real position of the scale. When he thinks he is looking at a scale on the wall the convergence is much less than when he knows that he is looking at a real card at $1 \mathrm{~m}$. distance. The difference between the two amounts of convergence indicates the extent of the psychological effect. A second card, half the size of that used at $1 \mathrm{~m}$., is now placed at $50 \mathrm{~cm}$., and the observation is repeated. Suppose this time that the line appears to be displaced $3^{\circ}$ to the left; the convergence for the line at zero will be $2 \times 3.7^{\circ}=7.4^{\circ}$, so that the actual convergence will be $7.4^{\circ}+3=10.4^{\circ}$.

The experiment is now repeated using cards of appropriate size placed at 33, 25 , and $20 \mathrm{~cm}$. distance, so that a card test is carried out to correspond with each of the $-1,-2,-3,-4$, and -5 dioptre lenses used in Test 1 . Visual acuity is checked for each position by a Snellen chart, reduced photographically by the correct amount, and placed by the card. It was found helpful to move the card sideways until the red line appeared to pass through it, for by this means it was possible to be certain that the observer was accommodating correctly while he was reading the position of the red line.

The results are shown graphically in Figs 2 and 3. Here there is no ambiguity in the terms esophoria and exophoria, and a point which lies above the orthophoric line represents an esophoria, while a point below it indicates an exophoric condition. It is useful here to notice that the values for the phorias obtained in this way with the card at $30 \mathrm{~cm}$., are approximately double those which are found using the Maddox wing. The reasons for this important difference are not understood. The value of the phoria at any distance may be read from the graph by noting the distance of the point above or below the orthophoric line.

\section{Test 3. (See key to Figs 2 and 3)}

The card used at $20 \mathrm{~cm}$. distance in the previous test is the only one used in this experiment; it is placed at $20 \mathrm{~cm}$. from the observer together with the appropriately reduced Snellen chart. The Maddox rod is placed as before in front of the left eye. A lens of $+5 \mathrm{D}$ is now added in front of the right eye and the observer is asked to read the chart. The image of the chart is at infinity, so if he reads it to Line 6 we can assume that the accommodation is relaxed. If the observer now states that the line is, say, $10^{\circ}$ to the right, we can calculate the actual convergence as follows:

If the visual axes were converged upon the lamp, the angle between them would be

$$
2 \times \tan ^{-1}\left(\frac{6.5}{2} \times \frac{1}{20}\right)=2 \times 9^{\circ}=18^{\circ} .
$$

The fact that the line is $10^{\circ}$ to the right indicates that he has converged by $10^{\circ}$ less than is required by the real position of the card; thus his actual convergence is $18^{\circ}-10=8^{\circ}$. The image of the card is at infinity, so that this represents an excess of convergence relative to accommodation. If the observer is orthophoric as shown by the normal Maddox rod test at $6 \mathrm{~m}$., we may deduce that the awareness of the nearness of the cards has induced a convergence of $8^{\circ}$.

After this lenses of $+4,+3,+2,+1$, zero, $-1,-2$, etc., dioptres are added until vision has deteriorated by one line on the chart. With, say, the +4D lens, the subject must accommodate by $+1 \mathrm{D}$ to make the $5 \mathrm{D}$ required for the card at $20 \mathrm{~cm}$; 位is is the accommodation normally required for an object at $1 \mathrm{~m}$., so that we may say that there is 
an image of the scale at $1 \mathrm{~m}$. For the other positive lenses images will be formed at 50,33 , and $25 \mathrm{~cm}$., while for zero lens the patient sees the real card at $20 \mathrm{~cm}$. Thus the card images coincide with the card positions for Test 2 and the difference between the amounts of convergence represent the influence of the proximal factor. That this influence is considerable is seen by the wide separation of the graphs of Tests 2 and 3 in Figs 2 and 3.

\section{Results}

Tests 1 and 2 for Normal Observers (Fig. 2).-As was expected, in most cases the amount of convergence was considerably greater when accommodation was induced by real cards being brought near (Test 2) than when it was caused by the addition of concave lenses. The surprising amount of variation between different observers resulted in their classification into three types:

(1) Accommodative.-The relation between accommodation and convergence is approximately the same whether the observer is looking at a virtual image or at a real card. An example is shown in Case 1 (Fig. 2). Only two individuals of this type were found, and strangely both were ophthalmic surgeons. This type is called "accommodative" because convergence appears to be induced solely by the accommodative effort, but a voluntary effort of convergence may also exist.

(2) Intermediate.-Some convergence is induced by the concave lenses in Test 1 , but there is a markedly greater amount in Test 2 using the real cards. Cases 2, 4, and 6 are examples of this category which was the most numerous.

(3) Perceptual.-No convergence at all is induced by the concave lenses, whereas the eyes converge considerably when real cards are brought near (e.g., Case 3). Here it seems that convergence is entirely determined by the perception of the remoteness of the wall.

Test 3 for Normal Observers (Fig. 2).-With the positive lenses and the card at $20 \mathrm{~cm}$., the amount of convergence is in all cases greater than in the corresponding portion of Test 2 , the increased convergence being no doubt induced by the awareness of the near position of the real card. Cases 3 and 5 show clearly how variable is the amount of the effect; thus in Case 3 the dotted line is nearly superimposed upon that for Test 2 for the greater part of its length, showing that for this observer the convergence is determined largely by the vergence of the light rays and the psychological effect is very small. By contrast, in Case 5, the dotted line departs widely from that of the full circles and for part of its course runs nearly parallel to the base line, showing that in these regions the estimate of the position of the object preponderates over the vergence clues. Just as in Test 1, some observers are influenced more by the perceived position of the object than by the accommodative effect, and tend towards the perceptual type, while in others the reverse is the case and so that they tend towards the accommodative type. It may be noticed that, as in Case 3, an individual may be a definite perceptual type for Test 1 (negative lenses), and yet tend to the accommodative type in Test 3 (positive lenses).

Tests 1 and 2 for Eye-Strain Group (Fig. 3).-A glance at the graphs of Cases $8,9,12,14,15,17$ reveals the important fact that perceptual types are very much more common in this group than in the normal group. 
The number of normal and asthenopic observers falling into each category is shown below:

\begin{tabular}{|c|c|c|c|c|}
\hline \multicolumn{3}{|c|}{ Observers } & Normal & Eye-Strain \\
\hline $\begin{array}{l}\text { Accommodative } \\
\text { Perceptual ... } \\
\text { Intermediate }\end{array}$ & $\begin{array}{l}\ldots \\
\ldots \\
\ldots\end{array}$ & $\begin{array}{ll}\ldots & \ldots \\
\ldots & \ldots \\
\ldots & \ldots\end{array}$ & $\left.\begin{array}{r}2 \\
5 \\
15\end{array}\right\} 22$ & $\left.\begin{array}{r|r}0 \\
11\end{array}\right\} 12$ \\
\hline
\end{tabular}

Test 3 for Eye-Strain Group (Fig. 3).-There was perhaps a tendency in this group for the proximal factor to predominate over the vergence clues to a greater extent than in the group of normals. There was however little correlation between Test 1 and Test 3, and Test 3 does not distinguish so clearly between normal and asthenopic groups as Test 1 .

\section{Discussion}

In discussing the problem of latent squint it is usually assumed that, when the stimulus to fusion is removed, the eyes will converge to an extent determined by the accommodative effort involved. The results given here show that this assumption is not true, and that the observer's estimate of the range of the object plays an all-important part. Furthermore, the mechanism of the near reflex varies astonishingly between different individuals. A complete range exists between the accommodative type, who converges as much in Test 1 (with concave lenses) as in Test 2 (with cards), and the perceptual type, who does not converge at all in Test 1 . It is remarkable that the complete range of psychological effect from 0-100 per cent. should be found in this very small sample of 22 normal people.

In a complaint described by the term " eye-strain", one would hardly expect a characteristic result from measurements of this kind, mainly because the symptoms are so often considered to be of mental origin. Consequently it is surprising to find how consistent are the graphed results of the affected group. All the members of this group except one (Case 12) belong to the perceptual type, and in Case 12 there was good reason to expect causes of eye-strain other than convergence defects, because this patient's eyes were constantly in motion, so much so that it was difficult to measure the interpupillary distance.

Case 9 was particularly interesting in showing that even in esophoria there may be a failure to increase the convergence when concave lenses are added. The patient was a vigorous male intellectual aged 40 , with no psychological symptoms, who complained that "eye-strain " made it hard for him to do the necessary amount of reading. This case should be compared with Case 4 , a vigorous female intellectual aged 36, in whom the esophoria (Test 2) is similar, but in whom there were no symptoms. Note the striking difference in the result of Test 1.

Case 17 is interesting because the esophoria at $30 \mathrm{~cm}$. of $5^{\circ}$ and $2^{\circ}$ 
(corresponding to approximately $5^{\Delta}$ and $2^{\Delta}$ on the Maddox wing) comes within normal limits. This was a case of convergence insufficiency.

By comparing the graphs of the normal observers with those of persons complaining of eye-strain, it appears that there are three factors which may contribute to the cause of this complaint: latent squint, absence of accommodative convergence (perceptual type), and low relative accommodation (short line in Test 2).

Test 3 shows little difference between eye-strain patients and normal observers, but the results are interesting. In all cases the awareness of the proximity of the object viewed caused a convergence which was excessive in relation to the accommodation demanded by the vergence of the light rays, and in many cases there was also a failure to relax the accommodation, an observation originally made by Landolt ? This effect is most striking, and it was amusing to see an experienced ophthalmic surgeon (Case 1) who had $6 / 6$ vision at $6 \mathrm{~m}$. without glasses, struggling to read the type on a card at $20 \mathrm{~cm}$. distance from him when given a $+5 \mathrm{D}$ lens. At first he complained that in view of his age he should not be asked to read type at such a short distance, but on being informed that the image was at infinity he was still unable to relax his accommodation and attained only 6/24 vision. Thus, in Case 1, since zero accommodation could not be obtained, this point is not plotted for Test 3 .

The results of Test 3 are very relevant to the prescription of reading spectacles. For instance, in Case 3, positive lenses affect convergence to a considerable extent, and to prescribe them for near work would certainly induce a latent squint. Since the observer is a pronounced perceptual type this would be likely to cause trouble. It is therefore very important to interpret the diagrams correctly, as is shown by the following example:

Suppose in Case 3 we consider the point on the graph defined by 3D accommodation and $11^{\circ}$ convergence. Near this point are found a triangle from the graph of Test 3 and a circle from the graph of Test 2 , both lying on the orthophoric line and nearly coinciding. In spite of this coincidence the points indicate totally different results in the two tests. A circle plotted at this point in Test 2 shows that there is no phoria when the Maddox rod test is performed with the light and scale at $33 \mathrm{~cm}$., but the triangle plotted above the abscissa value of 3D in Test 3 indicates that, of the 5D of accommodation required for an object at $20 \mathrm{~cm} ., 3 \mathrm{D}$ are contributed by the accommodative effort of the observer, so that this point corresponds to the use of a $+2 \mathrm{D}$ lens in the test. The fact that the triangle lies almost on the orthophoric line shows that the convergence is appropriate to the $3 \mathrm{D}$ of accommodation; but this is by no means appropriate to the distance of $20 \mathrm{~cm}$. at which the card is placed, a distance which demands a convergence of $19^{\circ}$ from this observer with a interpupillary distance of $65 \mathrm{~mm}$., so that the line appears displaced by $19-11$ $=8^{\circ}$; in fact, the observer announces this $8^{\circ}$, and by reversal of the above argument the point on the graph is plotted from it.

The work entailed in performing the three tests may take as long as 45 minutes even with an intelligent patient, and is not likely to be applicable clinically in its present form, but it seems likely that useful data could be obtained by making a much smaller number of readings. To simplify 
Test 1, a Maddox rod test could be made at $6 \mathrm{~m}$.; a pair of $-2 \mathrm{D}$ lenses is then inserted, and failure to converge will diagnose the perceptual type in nearly all cases. Test 3 could be simplified by carrying out a Maddox rod or prism diplopia test at $30 \mathrm{~cm}$., and then noting the effect upon convergence of placing, say, $a+2 D$ spherical lens in front of each eye.

In discussing the correction of presbyopia, Duke-Elder (1943) states that if too much of the available accommodation is replaced by over-strong glasses the relation between accommodation and convergence is upset, and that this is probably the most usual cause of the asthenopia and discomfort which may follow the correction of the complaint. It seems that the decrease of convergence occurring in the suggested simplified version of Test 3 would be a guide to the amount of correction that can be tolerated, and that when the decrease is considerable incorporation of base-in prisms should be considered.

\section{Summary}

It is usually assumed that when the fusion stimulus has been removed the amount of accommodative effort entirely determines the degree of convergence. It is here shown that this is not so, and that for most observers the conscious estimate of the position of an object (the proximal factor) plays a part at least as important as accommodation in determining convergence. The influence of this factor varies greatly from one individual to another.

Tests are described for measuring the effect at long and short distances. At long range, for nearly all asthenopic patients, the estimate of range predominates and prevents convergence, despite the accommodative effort induced by concave lenses; this is a result which could be applied to diagnosis. At short distances, the proximal factor affects the amount of latent squint induced by the wearing of positive lenses, and its measurement intimately concerns the appropriate correction for presbyopia and hypermetropia.

My sincere thanks are due to Mr. L. P. Jameson Evans for letting me examine his patients, to Mr. Frank Law for his help and advice, and to Miss Levinge of the Orthoptic Department of the Birmingham and Midland Eye Hospital for her willing co-operation.

\section{REFERENCES}

ASHER, H., and LAW, F. W. (1952). British Journal of Ophthalmology, 36, 225.

DUKe-ElDER, S. (1943). " The Practice of Refraction", 4th ed., p. 139. Churchill, London. HOFSTETTER, H. W. (1942). Amer. J. Optom., 19, 67.

ITtelson, W. H., and AMES, A. (1950). J. Psychol., 30, 43.

Morgan, M. W. (1944). Amer. J. Optom., 21, 183. 